\title{
Experiencia de aula basada en los enfoques de aprendizaje "estudiantes del grado décimo fomentan el desarrollo del espíritu científico en estudiantes de preescolar"1
}

\author{
Classroom experience based on learning approaches "tenth grade \\ students develop the scientific spirit in preschool students"
}

Experiência em sala de aula com base abordagens de aprendizagem

"estudantes décimo grau fomentar o desenvolvimento do espírito científico em estudantes pré-escolar"

Recibido: febrero de 2012 Aceptado: mayo de 2012
Manuel Guillermo Soler Contreras ${ }^{2}$ Adriana Moreno García ${ }^{3}$

\section{Resumen}

La experiencia se basa en un trabajo práctico orientado por los alumnos del grado décimo a un grupo de preescolar, para introducirlos al mundo de las ciencias experimentales. De este modo, los primeros, por medio de la metacognición, desarrollan competencias cognitivas, procedimentales y actitudinales, diseñando el material didáctico y la metodología apropiada paraimplementar, y los segundos, despiertan su espíritu científico.

Se reportan resultados que conducen a concluir que los alumnos de grado décimo, impulsados por una motivación intrínseca, han guiado su aprendizaje bajo un enfoque profundo, que los ha llevado a generar cambios conceptuales en relación a los temas abordados. Por su parte, los alumnos de preescolar, al verse orientados por sus compañeros de décimo, en una practica experimental que les genera tanto interés, han mostrado una actitud hacia la ciencia experimental que es necesario seguir cultivando.

Palabras clave: metacognición, trabajo práctico experimental, enfoque superficial, enfoque profundo, enseñanza, aprendizaje.

\begin{abstract}
The experience is based in a practical work oriented by the tenth grade students to a pre-school group in order to introduce them to the world of the experimental sciences. In this way the first trough out metacognition develop cognitive, procedural and attitudinal competences, designing the didactic material and the right methodology for implementing and the second encourage their scientific spirit.
\end{abstract}

1 Artículo de investigación. Presenta la experiencia que se ha realizado en el marco del proyecto de investigación sobre enfoques de enseñanza que adelanta el primer autor en la línea de investigación sobre evaluación, en el énfasis de ciencias, acciones y creencias del Doctorado Interinstitucional en Educación en la Universidad Pedagógica Nacional, con la valiosa colaboración de la segunda autora como especialista en educación preescolar.

2 Universidad Pedagógica Nacional de Colombia. Secretaría de Educación,Municipio de Soacha. Contacto: maguiso5@hotmail.com

3 Universidad de la Salle. Secretaría de Educación,Municipio de Soacha. Contacto: nenamorgar@hotmail.com 
The results are reported, and lead to conclude that the students from tenth grade, encouraged by an intrinsically motivation, have guided their learning under a deep approach that lead them to create conceptual changes in relation to the topics the deal with. On the other hand, the pre-school students have so much interest when they are guided by their tenth grades classmates; they also have shown an attitude towards the experimental science that is necessary to cultivate.

Keywords: metacogntion, experimental practical work, surface approach, deep approach, teaching, learning.

\section{Resumo}

A experiência é baseada em práticas de trabalho orientadas a estudantes do ensino fundamental (em idades entre 14 e 16 anos) para um grupo de estudantes de pré-escolar, para introduzi-los ao mundo das ciências experimentais. Assim, os estudantes do ensino fundamental através da metacognição desenvolvem habilidades cognitivas, procedimentais e atitudinais, além disso, com a criação de materiais didáticos e metodologia adequada para implementar, e os segundos despertam seu espírito científico

Os resultados levam à conclusão que os estudante do ensino fundamental impulsionado pela motivação intrínseca, têm orientado a sua aprendizagem em uma abordagem profunda, que os levou a produzir mudanças conceituais em relação aos temas discutidos. Os estudantes de pré-escolares ao ser dirigidos pelos colegas de ensino fundamental em uma prática experimental mui interessante, desenvolveram uma attitude na ciência experimental que é importante manter neles.

Palavras-chave: metacognição, trabalho prático experimental, a abordagem superficial, a abordagem profunda, ensino, aprendizagem.

\section{Introducción}

Dentro del amplio espectro de las líneas de investigación en didáctica de las ciencias, se ha escogido para la implementación de una estrategia metodológica en la enseñanza de algunos de sus contenidos, el uso de la metacognición, entendida como los procesos mentales que les permiten a los alumnos aprender a aprender. Es así que se plantea un programa de formación que gire alrededor de una actividad práctica experimental que involucre estudiantes de los grados décimoy prescolar. La estrategia persigue dos finalidades:de un lado, que los estudiantes de décimo grado, recurriendo a la metacognición, aprendan conceptos científicos enseñándolos a sus compañeros de prescolar; de otro lado, que los estudiantes de prescolar, motivados por sus compañeros de décimo, despierten un espíritu científico y una motivación intrínseca hacia la ciencia experimental.

Todo esto se sustenta con constructos teóricos como los enfoques de aprendizaje, los enfoques de enseñanza, la metacognición y el alineamiento constructivo, constructos desarrollados teóricamente para pisar en terreno firme a la hora de implementar la estrategia yrecolectar la información pertinente, y fruto de su análisis, extraer conclusiones que den cuenta de esta experiencia significativa.

Los detalles de la implementación de la experiencia son descritos con detalle soportando la información metodológica y los resultados en diagramas y tablas que conducen a deducciones plasmadas en el apartado de las conclusiones. Para no hacer 
denso el cuerpo del documento se ha optado por proporcionar el enlace de descarga ${ }^{28}$ de la guía "flota o se hunde" aplicada en esta estrategia, y en anexos algunos documentos de interés.

Esta experiencia se empezó a planificar en febrero de 2012, su implementación se dio durante el transcurso del segundo periodo académico (abril-mayo), el análisis de los resultados y la redacción del documento se ha dado entre junio y julio de 2012. Se proyecta continuar el proceso aprovechado los excelentes resultados obtenidos.

\section{Aspectos teóricos}

Desde el punto de vista del proceso de enseñanza y aprendizaje se han tomado como referentes de primera línea los planteamientos de investigadores de las escuelas de Edimburgo y Gotemburgo, liderados por Marton y Säljö en Suecia (Säljö y Marton, 1976a, 1976b), Entwistle, Hanley, Hounsell y Ramsden en Inglaterra (Entwistle, 1987) y su continuación por John Biggs en Hong Kong y Australia, en relación a los enfoques de aprendizaje; y por un lado, de Kember y Gow en Hong Kong (Kember y Gow, 1993), y por el otro, Trigwell y Prosser en Londres (Trigwell, Prossery Waterhouse, 1999)en relación a los enfoques de enseñanza. Estas líneas de investigación se han ido extendiendo a países de habla hispana como España y países latinoamericanos (Porto, 1994 y Hernandez Pina, 1993).

Los enfoques de aprendizaje han sido definidos por Biggs como "los procesos de aprendizaje que emergen de las percepciones que los estudiantes tienen de las tareas académicas, influidas por sus características de tipo personal" (Biggs, 2005), es decir, el significado no se impone ni se transmite mediante la enseñanza directa, sino que se crea mediante las actividades de aprendizaje. Lo que construyen las personas a partir de un proceso de aprendizaje depende de sus motivos e intenciones, de lo que saben y de cómo utilicen sus conocimientos previos.

Los enfoques de aprendizaje están en función tanto de las características individuales de los alumnos, como del contexto de enseñanza determinado. Por esta razón, un enfoque de aprendizaje describe la naturaleza de la relación entre alumno, contexto y tarea (Soler, 2012).

Desde la perspectiva de los enfoques de aprendizaje, el trabajo que aquí se presenta pretende encaminar a los estudiantes de grado décimo para que, por medio de procesos metacognitivos elijan una ruta de aprendizaje orientada bajo el enfoque profundo, es decir, generarles una motivación intrínseca que los lleve a plantear estrategias basadas en la metacognición, para enseñar ciertos principios básicos de las ciencias experimentales a sus compañeros de prescolar, de modo que les despierteninterés por las ciencias naturales.

Mediante esta experiencia se persigue lograr implementar lo que Biggs (2005) ha llamado el alineamiento constructivo, el cual consiste en alinear todas las fases de un proceso educativo, esquematizado por este autor en el modelo $3 \mathrm{P}$ - presagio, proceso y producto-, la variable de presagio incluye las ideas previas de los estudiantes, el clima de la clase y todo el aspecto contextual; la variable proceso hace referencia a las rutas de aprendizaje elegidas por los estudiantes y a la naturaleza de la tarea a desarrollar, es decir, al enfoque de aprendizaje de los estudiantes y al enfoque de enseñanza de los profesores (Trigwell, Prosser y Ginns, 2005); la variable producto se refiere a los resultados de procesos de formación emanados por los distintos modos de evaluación utilizados.

En cuanto a la metacognición como estrategia para aprender ciencias, ésta es una de las líneas de investigación de mayor auge en las dos últimas décadas, es por ello que abundan las publicaciones de reconocidos autores que han tenido predilección por esta línea en sus estudios. De todo este cúmulo de trabajos se ha recurrido a uno realizado por Campanario (2000) que lleva por título El desarrollo de la metacognición en el aprendizaje de las ciencias: Estrategias para el profesor y actividades orientadas al alumno, como el título lo sugiere, en este documento, el autor se da a la tarea de sugerir

4 Taller descargado en enero de 2012 y disponible en: http://www.concyteq.edu.mx/PDF/ManualPreescolarUltimaVersion.pdf 
una serie de siete estrategias para el profesor y once actividades orientadas al alumno, las mismas se relacionan en la tabla 1.

\begin{tabular}{|c|c|}
\hline Estratégias para el profesor & $\begin{array}{l}\text { Actividades orientadas al } \\
\text { alumno }\end{array}$ \\
\hline $\begin{array}{l}\text { Programas explícitos de } \\
\text { instrucción }\end{array}$ & $\begin{array}{l}\text { Actividades: } \\
\text { predecir-observar-explicar }\end{array}$ \\
\hline $\begin{array}{l}\text { Dar a conocer los obje- } \\
\text { tivos del proceso de } \\
\text { enseñanza-aprendizaje }\end{array}$ & Mapas conceptuales \\
\hline $\begin{array}{l}\text { Insistir en el compo- } \\
\text { nente problemático del } \\
\text { conocimiento }\end{array}$ & Diagramas V \\
\hline $\begin{array}{l}\text { Aplicación de los conoci- } \\
\text { mientos científicos a la reali- } \\
\text { dad cotidiana }\end{array}$ & $\begin{array}{l}\text { Uso adecuado de la } \\
\text { bibliografía }\end{array}$ \\
\hline $\begin{array}{l}\text { El recurso a la historia de la } \\
\text { ciencia }\end{array}$ & $\begin{array}{l}\text { Resolución de problemas como } \\
\text { pequeñas investigaciones }\end{array}$ \\
\hline $\begin{array}{l}\text { El desarrollo de enfoques } \\
\text { multidisciplinares }\end{array}$ & $\begin{array}{l}\text { Resolución de problemas con } \\
\text { soluciones contraintuitivas }\end{array}$ \\
\hline \multirow[t]{5}{*}{$\begin{array}{l}\text { Uso de la evaluación como } \\
\text { instrumento metacognitivo }\end{array}$} & $\begin{array}{l}\text { Realización de actividades de } \\
\text { materialización }\end{array}$ \\
\hline & Elaboración de un diario \\
\hline & Empleo de autocuestionarios \\
\hline & $\begin{array}{l}\text { Preguntas cortas para contes- } \\
\text { tar por escrito }\end{array}$ \\
\hline & $\begin{array}{l}\text { Formulación de preguntas por } \\
\text { parte de los propios alumnos }\end{array}$ \\
\hline
\end{tabular}

Tabla 1. Estrategias para el profesor y actividades orientadas al alumno

Fuente: elaboración propia

En calidad de docentes investigadores, hemos hecho uso de la primera y la séptima estrategia relacionadas en la tabla anterior relativas al docente de la siguiente manera:

Programas explícitos de instrucción: la metodología muestra cómo se han asignado a grupos de estudiantes de décimo tareas de tipo ejecutivo para liderar procesos de formación en alumnos de prescolar por medio de actividades prácticas experimentales. Se han aceptado las sugerencias Campanario (2000) en cuanto a los componentes de este tipo de programas como sigue:

- Introducción general: explicación del propósito de la estrategia anticipando las ventajas de realizarla y los conceptos que van implícitos.
- Ejemplos de la actividad metacognitiva: se ilustra mediante variados ejemplos el proceso de implementación de la estrategia.

- Explicación: demostración activa de la estrategia incentivando la participación de todos los estudiantes, el profesor sirve como modelo para los alumnos.

- Practica dirigida: bajo el control del profesor se realiza la aplicación de la estrategia.

- Practica independiente: se abre el espacio para que el alumno realice la experiencia de forma independiente, colaborativa y autorregula

En cuanto a las actividades orientadas al alumno se ha echado mano a las actividades predecir-observar-explicar: mediante estas tres etapas los estudiantes hacen uso de sus ideas previas, las ponen a prueba y generan un cambio conceptual durante la aplicación de la estrategia metacognitiva, pues para poder explicar la experiencia a un niño de preescolar ellos ven la necesidad de apropiar los conceptos de la forma científicamente aceptada de manera universal.

En relación al proceso de aprendizaje de los niños, se ha tomado como soporte teórico la psicología evolutiva o genética de Jean Piaget, quien describe unos periodos de desarrollo del niño mediados por la interacción de sus estructuras mentales con el ambiente. Para efectos de este trabajo interesa analizar el periodo de desarrollo entre los dos y siete años, en donde el niño descubre que algunas cosas pueden tomar el lugar de otras. El pensamiento infantil ya no depende de acciones únicamente externas, sino que también se interioriza (Piaget, 1978). Se desarrollan habilidades tales como la imitación, el juego simbólico, la imagen mental y un rápido desarrollo del lenguaje hablado.

También se encuentran algunos obstáculos como la incapacidad de intervenir mentalmente una acción física para regresar un objeto a su estado original (reversibilidad), incapacidad de retener mentalmente cambios en dos dimensiones al mismo tiempo (centración), incapacidad para tomar en cuenta otros puntos de vista (egocentrismo). Estos aspectos piagetianos han sido tenidos en cuenta a la hora de planificar la experiencia significativa aquí reportada. 


\section{Metodología}

La experiencia consiste en realizar con los estudiantes de preescolar una práctica experimental orientada por los estudiantes del grado décimo bajo la tutela de los docentes investigadores de los correspondientes cursos, con el propósito de fomentar la formación del espíritu científico en los primeros y afianzar aspectos conceptuales en los segundos, por medio de estrategias metacognitivas. La tabla 2 muestra las características de los grupos que intervienen en la experiencia:

\begin{tabular}{|l|l|l|}
\hline \multicolumn{3}{|l|}{ Caracterización de los grupos } \\
\hline Alumnos & Décimo & Preescolar \\
\hline Mujeres & 19 & 13 \\
\hline Hombres & 24 & 17 \\
\hline Total & 43 & 30 \\
\hline Edad (años) & $14-16$ & $5-6$ \\
\hline
\end{tabular}

Tabla 2. Descripción de los grupos que intervienen el la experiencia significativa.

Fuente: elaboración propia

Se ha seleccionado, dentro de muchas opciones, un trabajo práctico experimental que lleva por nombre "flota o se hunde" dada su sencillez en cuanto a materiales usados, al procedimiento a seguir, y ante todo a la riqueza de conceptos que lleva implícita y que ameritan un cambio conceptual tanto en los alumnos del grado décimo como en los del grado preescolar, en relación al presupuesto de ideas previas que, con respecto a dichos temas, ellos traen.

Por lo anterior, el primer paso ha sido la indagación de estas preconcepciones en estos dos grupos focales. Para ello, los docentes investigadores han diseñado un instrumento para aplicarlo: de forma escrita a los alumnos de grado décimo, y a modo de entrevista a los alumnos del grado preescolar. Se asignó la actividad de consultar una definición científica de cada concepto que involucra la práctica experimental, para luego, en plenaria, discutir estas definiciones y establecer puestas en común. En el apartado de resultados (ver tablas 3 y 4 ) se reportan las ideas previas identificadas para cada concepto en los grupos implicados en la investigación.
El segundo paso consistió en realizar la práctica en el grado décimo, permitiendo que los estudiantes falseen las hipótesis que previamente han postulado, también que tomen atenta nota en relación a los interrogantes que han surgido de la misma.

Como tercer paso, se ha dividido el grupo de décimo en diez grupos de cuatro estudiantes y el de preescolar en diez grupos de tres estudiantes, para realizar la correspondiente asignación. En esta sesión se asignaron responsabilidades a cada grupo de décimo, como el diseño de material didáctico de acuerdo a las indicaciones, la consecución del material de laboratorio necesario para realizar la práctica con el grupo de preescolar - material casero de fácil adquisición, de sencilla manipulación y que no acarrea ningún tipo de riesgo para los estudiantes-. Se ha propiciado también un debate en relación a los interrogantes que la realización de la práctica experimental ha suscitado, también se ha puesto especial énfasis en dejar claro que el proceso de aprendizaje de los alumnos de preescolar, en esta práctica, es responsabilidad total de ellos, por lo que se dieron indicaciones en cuanto al lenguaje a utilizar y al diseño del material.

El cuarto paso consistió en realizar la practica experimental. Cada grupo de preescolar es orientado por un grupo de décimo bajo la orientación de los docentes investigadores.

Finalmente, en elquinto paso, cada docente investigador en sesión con su respectivo grupo, verifica el cambio conceptual generado como producto de esta experiencia. En preescolar, por medio de la expresión gráfica, la docente ha constatado lo pertinente. Por su parte, en décimo se ha verificado una de las premisas de la metacognición en cuanto a que la mejor forma para que un estudiante pueda verificar por sí mismo su grado de comprensión, es poniéndose a prueba, intentando explicar los conceptos a compañeros de su grado o de grados inferiores, tomando el rol de tutor y asegurándose de que su discípulo entienda.

\section{Resultados}

Los resultados del diagnóstico de los conocimientos previos a la experiencia de laboratorio "flota o se hunde" se relacionan en la tabla 3 . 


\section{- CONOCIMIENTOS PREVIOS}

Indagar sobre los conocimientos previos que tienen los estudiantes del grado preescolar 2 sobre los siguientes conceptos: liquido, volumen, flotar, hundir, peso, fuerza, disolver-

La noción conceptual de los estudiantes se evidencia de la siguiente manera sin contextualizarlo con la experiencia del laboratorio: LIQUIDO: para lavar, no sabe, es aceite, tomar, no sabe, gaseosa, agua, colbón, agua, agua, para lavar, algo que se intoxica, gasolina, agua, agua.

VOLUMEN: escuchar música, le sube mucho, subir al televisor, hay muchos gritos, escuchar música, subir el volumen, escuchar, que no le sube a algo, es muy alto, suben mucho, que uno sube, que uno sube volumen a todo, subir a un radio y al televisor, mucho ruido, subir el volumen.

DISOLVER: resolver algo, silvar, hacer la tarea, no lo sabe, no lo sabe, mirar, que devuelve algo, es agua, no lo sabe, no lo sé, resuelve un misterio, agua, que se toman toda el agua, que todo se resuelve.

FLOTAR: cuando va a piscina y se pone a flotar, volar, flotar en el agua, flotar en los barcos, cuando se quema algo y se flota, que flota en una bomba, que flota una bomba, que tiene algo para que no se hunda, flota en una cosa como agua, que uno flota, que flota por el agua, que flota por el aire, que flota en el agua.

HUNDIR: no hay un salvavidas, caer, se hunde las casas, no lo sé, no lo sé, que se hunde en una piscina, hundir en una piscina, que no está hasta el fondo, como si alguien se está escondiendo, que algo se hunde, que algo se hunde, hundirse en la piscina, que se caen las cosas.

PESO: carga un bulto de arena abono químico donde trabaja mi papá, pesa, pesa, que uno no puede levantar las cosas, no sé, un peso, pez, que hace ejercicio para tener fuerza, algo que tuviera peso, que uno esta muy pesado, que tiene mucho peso, que pesa mucho.

FUERZA: trabaja algo pesado y fuerte, una gente que hace fuerza, músculos, para levantar cosas, hacer ejercicio, hacer ejercicio, un hombre que tiene fuerza, que hace mucho ejercicio, levanta algo que es pesado, que uno tiene mucha fuerza, que tiene mucha fuerza, levantar una cosa, que tiene mucha fuerza, tener mucha fuerza.

Tabla 3. Identificación de los conocimientos previos de los niños de preescolar.

Fuente: elaboración propia

La tabla 4 relaciona las ideas previas predominantes en los alumnos del grado décimo identificadas en la actividad diagnóstica.

\begin{tabular}{|c|c|c|}
\hline Conceptos & leas previas & Términos frecuentemente utilizados en relación al concepto \\
\hline Flotar & \multirow{8}{*}{$\begin{array}{l}\text { Los alumnos de décimo recurren cons- } \\
\text { tantemente a términos del lenguaje } \\
\text { común al intentar explicar cada tér- } \\
\text { mino. También establecen relaciones } \\
\text { con otros conceptos científicos de } \\
\text { los cuales tienen ideas previas mas } \\
\text { fundamentadas. Una constante es } \\
\text { que suelen entrar en redundancias } \\
\text { como: "flotar es flotar en el agua", "el } \\
\text { peso es la densidad de un cuerpo". } \\
\text { Algunos redactan párrafos bien ela- } \\
\text { borados y argumentados que dan } \\
\text { cuenta de la claridad que tienen } \\
\text { del concepto. Muchos dejan sin res- } \\
\text { puesta a algunos de los términos. }\end{array}$} & $\begin{array}{l}\text { Peso, densidad, superficie, globo, gravedad, elevarse, no tocar el fondo, masa, } \\
\text { estar encima, liviano. }\end{array}$ \\
\hline Hundir & & Estar en el fondo, peso, descender, densidad, masa, volumen. \\
\hline Gravedad & & $\begin{array}{l}\text { Oxígeno, poca masa, igual posición, tiempo que tarda en caer algo, poco mag- } \\
\text { netismo, cuando algo flota debido al peso, llo que ayuda a hundir, energia que } \\
\text { mueve todo. }\end{array}$ \\
\hline Densidad & & $\begin{array}{l}\text { Es el peso, es el volumen, depende de la forma, fuerza de los elementos, es el } \\
\text { peso molecular, cantidad de masa, es todo lo grande. }\end{array}$ \\
\hline Peso & & $\begin{array}{l}\text { Cantidad de masa, toda la materia de un cuerpo, fuerza constante de una cosa, } \\
\text { cantidad de volumen, es la densidad de una cosa. }\end{array}$ \\
\hline Volumen & & $\begin{array}{l}\text { Lo grande de un cuerpo, es lo ancho y largo de un cuerpo, tamaño de una masa, } \\
\text { es lo que tiene una cosa, aumento de la masa y el peso, cantidad de masa que } \\
\text { hace que aumente el tamaño. }\end{array}$ \\
\hline Empuje & & Fuerza sobre algo, mover algo, correr algo, es dar un golpe, \\
\hline Disolver & & $\begin{array}{l}\text { Fusión de dos cosas, desintegrar en el agua, reacción de un cuerpo frente a un } \\
\text { líquido, mezclar cosas, revolver, diluir algo sólido en algo líquido, revolver en } \\
\text { algo caliente, echar una sustancia en agua, efecto de fundir. }\end{array}$ \\
\hline
\end{tabular}

Tabla 4. Identificación de ideas previas en alumnos del grado décimo.

Fuente: elaboración propia 
La tabla 5 relaciona las principales observaciones en cada una de las fases de la implementación de la actividad práctica experimental.

\section{- PERCEPCIÓN VERBAL DEL LABORATORIO}

Permitir por medio de un video, después del laboratorio, evidenciar la percepción verbal de los estudiantes respecto a los nuevos conceptos

En el video se evidencia cómo algunos estudiantes se expresan en un lenguaje espontaneo, como de forma secuencial, se realizó la experiencia de laboratorio "flota o se hunde", utilizando términos como subir y bajar, o subir cuando flota y bajar cuando se hunde.

\section{- ANÁLISIS DE LA REPRESENTACIÓN GRÁFICA}

Por medio del dibujo con plumones permitir que los estudiantes representen la experiencia significativa del laboratorio y realizar un análisis.

La representación gráfica se evidencia de la siguiente forma:

- 29 niños realizaron la representación grafica.

- 19 niños realizaron una representación de la noción conceptual de la experiencia de laboratorio "flota o se hunde".

- 10 Niños realizaron una representación de la Experiencia de laboratorio

-

\begin{tabular}{|l|l|}
\hline $\begin{array}{l}\text { REPRESENTACIÓN DE LA NOCIÓN CONCEPTUAL } \\
\text { DE LA EXPERIENCIA DE LABORATORIO “FLOTA O SE } \\
\text { HUNDE" }\end{array}$ & $\begin{array}{l}\text { REPRESENTACIÓN DE LA EXPERIENCIA DE } \\
\text { LABORATORIO “FLOTA O SE HUNDE" }\end{array}$ \\
\hline $\begin{array}{l}\text { En los } 19 \text { dibujos que realizaron los niños se ve } \\
\text { representado específicamente los recipientes y la } \\
\text { ubicación espacial del huevo }\end{array}$ & $\begin{array}{l}\text { En los } 9 \text { dibujos que realizaron los niños y las } \\
\text { niñas se ve representada la experiencia de } \\
\text { laboratorio. En la relación con los estudiantes } \\
\text { del grado } 1002 \\
\text { Un solo niño represento como en diferentes } \\
\text { situaciones se hunde y flota las personas. }\end{array}$ \\
\hline
\end{tabular}

Tabla 5. Percepción verbal y representación gráfica.

Fuente: elaboración propia

La tabla 6 relaciona los principales hallazgos encontradosdurantela realización dela experiencia significativa.

\begin{tabular}{|c|c|c|c|c|}
\hline & Fase & Descripción & Observaciones décimo & Observaciones preescolar \\
\hline$\widetilde{d}$ & \multirow[b]{2}{*}{ Primer momento } & $\begin{array}{l}\text { Presentación de los grupos de } \\
\text { trabajo: los alumnos de décimo se } \\
\text { presentaron y pidieron a los de pre- } \\
\text { escolar que también lo hicieran. }\end{array}$ & $\begin{array}{l}\text { Se distingue en cada grupo asigna- } \\
\text { ción de diferentes roles de liderazgo: } \\
\text { tutor, instrumentador y secretario. }\end{array}$ & $\begin{array}{l}\text { Se percibe los diferentes tipos } \\
\text { de personalidad de los niños: el } \\
\text { retraído, el hiperactivo, el atento, el } \\
\text { curioso. }\end{array}$ \\
\hline 미 & & $\begin{array}{l}\text { Descripción del material: los alum- } \\
\text { nos de décimo presentaron el mate- } \\
\text { rial que se utiliza en la experiencia } \\
\text { describiendo la función que cumple } \\
\text { dentro de la experiencia. }\end{array}$ & $\begin{array}{l}\text { Se nota apropiación de los princi- } \\
\text { pios de funcionamiento de cada } \\
\text { uno de los materiales. }\end{array}$ & $\begin{array}{l}\text { La expectativa es grande, los niños } \\
\text { hacen muchas preguntas, quieren } \\
\text { saberlo todo, quieren manipular cada } \\
\text { cosa, y pretenden anticipar lo que se } \\
\text { va a hacer. }\end{array}$ \\
\hline \multirow{2}{*}{ 它 } & \multirow[t]{2}{*}{$\begin{array}{l}\text { Segundo } \\
\text { momento }\end{array}$} & $\begin{array}{l}\text { Presentación del material didáctico: } \\
\text { cada grupo utilizó el material dise- } \\
\text { ñado para hacer una introducción } \\
\text { de la práctica y generar motivación } \\
\text { y expectativa hacia la misma. }\end{array}$ & \multirow[t]{2}{*}{$\begin{array}{l}\text { Se ha hecho apropiado uso del } \\
\text { material diseñado, aquí los alumnos } \\
\text { han sido muy creativos tanto en el } \\
\text { diseño como en su uso pedagógico. }\end{array}$} & \multirow{2}{*}{$\begin{array}{l}\text { La atención prestada es muy satis- } \\
\text { factoria, hacen muchas preguntas } \\
\text { y predicciones de lo que ocurrirá } \\
\text { usando lenguaje que no se esperaba } \\
\text {-el huevo se hunde por que no } \\
\text { sabe nadar, el huevo queda salado, } \\
\text { el huevo queda dulce--. }\end{array}$} \\
\hline & & $\begin{array}{l}\text { Se plantearon preguntas para que } \\
\text { los niños predijeran los resultados } \\
\text { que se obtendrían. }\end{array}$ & & \\
\hline
\end{tabular}


EXPERIENCIA DE AULA BASADA EN LOS ENFOQUES DE APRENDIZAJE "ESTUDIANTES DEL GRADO DÉCIMO FOMENTAN EL DESARROLLO DEL ESPÍRITU CIENTÍFICO EN ESTUDIANTES DE PREESCOLAR"

\begin{tabular}{|l|l|l|l|}
\hline \multirow{5}{*}{ Tercer momento } & $\begin{array}{l}\text { Realización de la actividad práctica } \\
\text { experimental: los grupos pusieron } \\
\text { en marcha la práctica, haciendo que } \\
\text { los niños participaran activamente } \\
\text { en la misma. }\end{array}$ & $\begin{array}{l}\text { Se mostradon competencias } \\
\text { procedimentales y actitudinales } \\
\text { de alta calidad. La capacidad de } \\
\text { observación tanto de los aspectos } \\
\text { experimentales como de los com- } \\
\text { portamentales de los niños ha sido } \\
\text { muy buena. }\end{array}$ & $\begin{array}{l}\text { Todos los niños quieren participar } \\
\text { activamente, desean manipular } \\
\text { todo y lanzan hipótesis sobre lo que } \\
\text { sucederá. }\end{array}$ \\
\cline { 2 - 3 } $\begin{array}{l}\text { Contrastar con lo predicho: los } \\
\text { alumnos de décimo ibancontras- } \\
\text { tando los resultados con lo predicho }\end{array}$ & $\begin{array}{l}\text { Justificar resultados: los alumnos de } \\
\text { décimo utilizando de nuevo el mate- } \\
\text { rial didáctico instaron a los alumnos } \\
\text { de preescolar a que contrastaran los } \\
\text { resultados con lo predicho y sugirie- } \\
\text { ran explicaciones. }\end{array}$ & $\begin{array}{l}\text { Los alumnos pusieron a prueba su } \\
\text { imaginación para diseñar activi- } \\
\text { dades lúdicas que les permitiera } \\
\text { en complemento con el material } \\
\text { didáctico afianzar los conceptos } \\
\text { demostrados. }\end{array}$ & $\begin{array}{l}\text { Los alumnos se involucraron con } \\
\text { entusiasmo en las actividades lúdi- } \\
\text { cas planteadas por sus compañeros } \\
\text { de décimo. }\end{array}$ \\
\hline
\end{tabular}

Tabla 6. Descripción de los resultados en cada fase de la implementación de la actividad práctica experimental.

Fuente: elaboración propia

\section{Constatación de noción conceptual adquirida en los niños de preescolar}

Constatar los aprendizajes adquiridos en una nueva experiencia donde los estudiantes de preescolar la realicen.

En esta segunda experiencia se evidencia cómo la gran mayoría de los niños logran apropiarse de la noción conceptual flotar y hundir en el momento en el que se realiza la experiencia de laboratorio. Solo un niño hace referencia a subir y bajar.

\section{Conclusiones}

Las estrategias de enseñanza-aprendizaje fundamentadas en la metacognición, como la aquí socializada, generan en los estudiantes una motivación intrínseca que los impulsa a guiar su aprendizaje bajo un enfoque profundo. Los alumnos de grado décimo asumieron un reto y despertaron un sentido de responsabilidad no manifestado con anterioridad, esto, dado que se ven ante el compromiso de fomentar el espíritu científico en sus compañeros de prescolar.

La calidad de los recursos didácticos diseñados por losestudiantes de décimo ha sido excelente, no solo en lo estético, también en la originalidad y rigurosidad conceptual. Pero más importante aún es el buen uso que han hecho del mismo, esto se ha visto reflejado en el gran interés mostrado por los estudiantes de preescolar en el desarrollo de la práctica experimental (ver anexo2).
Los estudiantes de prescolar se han acercado a las ciencias experimentales de una forma amable, se han mostrado muy interesados y motivados y se ha potenciado su curiosidad gracias a la adecuada orientación de sus compañeros de décimo grado. Los niños de prescolar han desarrollado niveles de competencia de la siguiente manera:

- A nivel cognitivo:

Todos los estudiantes evidenciaron de forma verbal la noción conceptual de la experiencia de laboratorio "flotar o hundir" aunque aún hay algunos que no alcanzaron la abstracción de los factores concretos en el momento de la representación gráfica.

- A nivel procedimental:

Se logra demostrar de forma verbal y grafica (ver anexo 3) la secuenciación de la experiencia a nivel de noción conceptual por parte de algunos niños y en otros sólo se evidenció la representación de la experiencia.

- A nivel actitudinal:

Se evidenció, durante los dos momentos de la experiencia, gran interés y motivación por parte de los estudiantes, como en la interacción con los estudiantes del grado décimo. Es notable la claridad conceptual que los alumnos de décimo muestran luego de haber implementado la experiencia, en comparación con lo diagnosticado en la primera fase de este proceso de formación. Finalmente, se resalta la capacidad 
de observación de los estudiantes de décimo no solo en el aspecto experimental sino también en el reporte de esta actividad en el formato diseñado para este fin (ver anexo 1), allí pusieron de manifiesto competencias comunicativas importantes en relación a la escritura.

\section{Referencias}

Biggs, J. (2005). Calidad del aprendizaje universitario. Madrid: Nacea.

Campanario, J. (2000). "El desarrollo de la metacognición en el aprendizaje de las ciencias: Estrategias para el profesor y actividades orientadas al alumno". Investigación Didáctica.369-380.

Entwistle, N. (1987). La comprensión del aprendizaje en el aula. Madrid: Paidos.

Hernandez Pina, F. (1993). "Concepciones en el estudio del aprendizaje de los estudiantes universitarios". Revista de Investigación Educativa. (22).

Kember, D.and Gow, L. (1993). "Conceptions of teaching an teir relationship to student learning". British Journal of Educational Psychology. (63): 20-23.

Marton, F.and Säljö, R. (1976a). “On qualitative differences in learning: 1. Outcome and process". British Journal of Educational Psychology. (46): 4-11.

Marton, F.and Säljö, R. (1976b). "On quialitative differences in learning: 2. Outcome as a function of the leamer's conception of the task". British Journal of Educational Psychology. 115-217.

Piaget, J. (1978). La equilibración de las estructuras cognitivas, "Problema central del desarrollo”. Madrid: Siglo XXI.

Porto, A. (1994). Las aproximaciones al proceso de aprendizaje en estudiantes universitarios. (Disertación doctoral inédita). Santiago de Compostela.

Prosser, M.and Trugwell, K. (2006). “Conformatory factor analysis of the Approaches to
Teaching Inventory". British Journal of educational Psychology. (76): 405-419.

Ramsden, P. (1992). Learning to teach in Higher Education. Londres: Routlege.

Soler, C. M. (2012). El pensamiento de John Biggs "algunos elementos teóricos acerca del alineamiento constructivo y los enfoques de aprendizaje. En: Memorias III congreso internacional de investigación en educación, pedagogía y formación docente.

Trigwell, K., Prosser, M. and Ginns, P. (2005). "Phenomenographic pedagogy and a revised approaches to Teaching Inventory". Higher Education Research \& Development. 24(4): 349-360.

Trigwell, K., Prosser, M. and Waterhouse, F. (1999). "Relations between teachers'approaches to teaching and students'approaches to learning". Higher Education. (37): 57 - 70.

\section{Anexos}

\section{Anexo 1. Instrumento de seguimiento para grado décimo}

EXPERIENCIA DE LABORATORIO "FLOTA O SE HUNDE"

\section{PREESCOLAR 2}

JORNADA: MAÑANA

\section{Lic. ADRIANA MORENO GARCIA}

\section{Lic. MANUEL GUILLERMO SOLER CONTRERAS}

Actividad: indagar en la experiencia de laboratorio "Flota o se hunde" como se evidencia el espíritu científico en los estudiantes del grado preescolar 2 de la jornada mañana, en colaboración con los estudiantes del grado 1002 
EXPERIENCIA DE AULA BASADA EN LOS ENFOQUES DE APRENDIZAJE "ESTUDIANTES DEL GRADO DÉCIMO FOMENTAN EL DESARROLLO DEL ESPÍRITU CIENTÍFICO EN ESTUDIANTES DE PREESCOLAR"

INTEGRANTE GRADO 1002

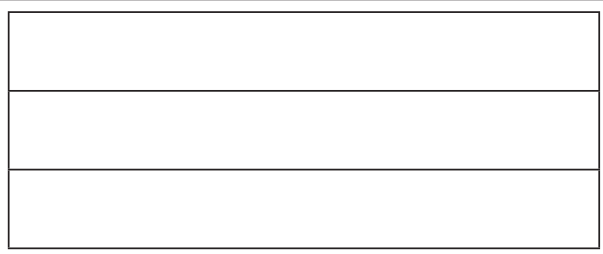

INTEGRANTES PREESCOLAR 2

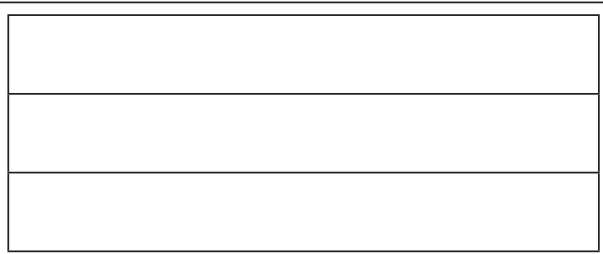

1. De forma descriptiva escriba quéocurrió en el primer momento de la experiencia de laboratorio "flota o se hunde"

2. De forma descriptiva escriba quéocurrió en el segundo momento de la experiencia de laboratorio "flota o se hunde".

3. De forma descriptiva escriba quéocurrió en el tercer momento de la experiencia de laboratorio "flota o se hunde".

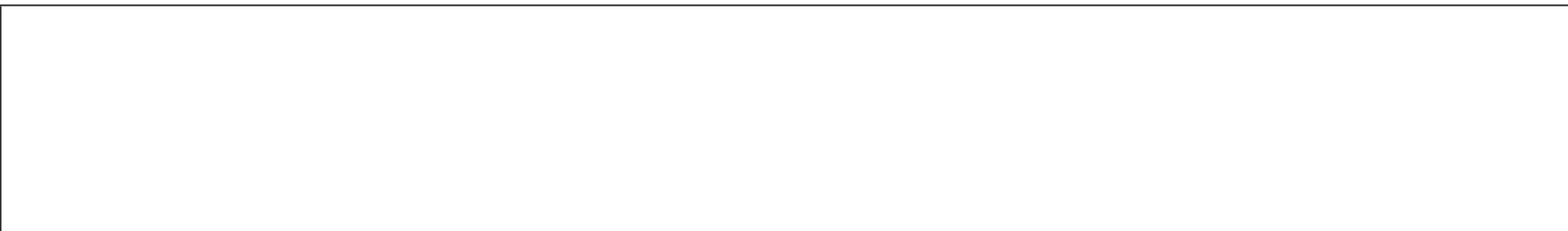

REVISTA CIENTÍFICA / ISSN 0124 2253/ JULIO- DICIEMBRE DE 2012 / No. 16 / BOGOTÁ, D.C. 
MANUEL GUILLERMO SOLER CONTRERAS, ADRIANA MORENO GARCÍA

4. ¿Ustedes piensan que ocurrió un cuarto momento en la experiencia de laboratorio "flota o se hunde"?. Argumentar.

5. Escriba todas y cada una de las expresiones manifestadas por los niños de preescolar 2 durante la experiencia "flota o se hunde".

6. ¿Qué estrategias didácticas utilizaron durante la experiencia de laboratorio "flota o se hunde"?

7. En cada uno de los cuadros cada integrante deberá escribir, cómo se apropiódel laboratorio "flota o se hunde" y logróenseñarlo a los estudiantes del grado preescolar 2.

\begin{tabular}{|l|l|l|}
\hline NOMBRE: & NOMBRE: \\
\cline { 2 - 4 } & NOMBRE: & NOMBRE: \\
\cline { 2 - 4 } & NOMBRE: & NOMBRE: \\
\hline
\end{tabular}


Anexo 2. Material didáctico diseñado por los estudiantes del grado décimo e implementación de la estrategia metodológica en preescolar.

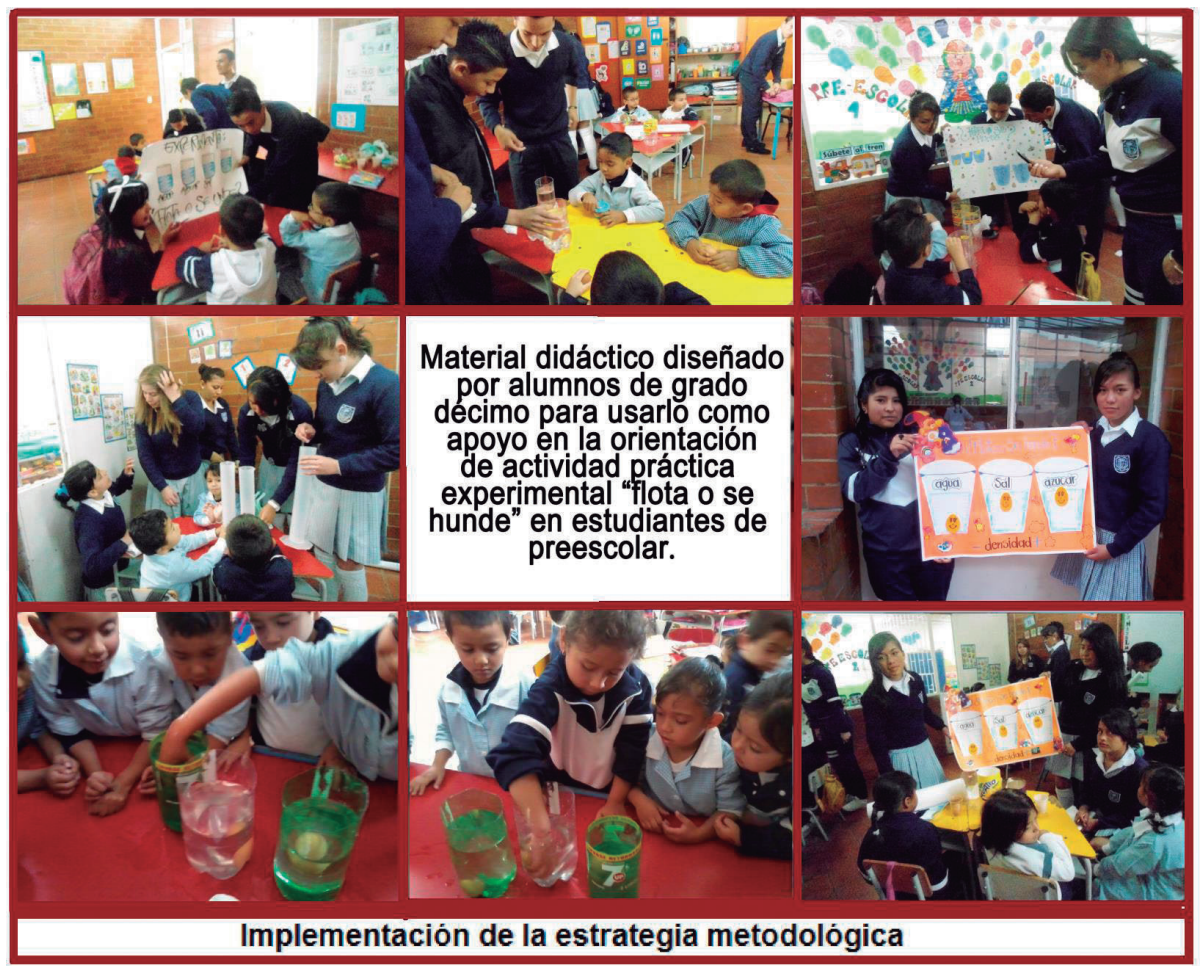

Anexo 3. Representación gráfica de la experienica de laboratorio hecha por los estudiantes de preescolar.

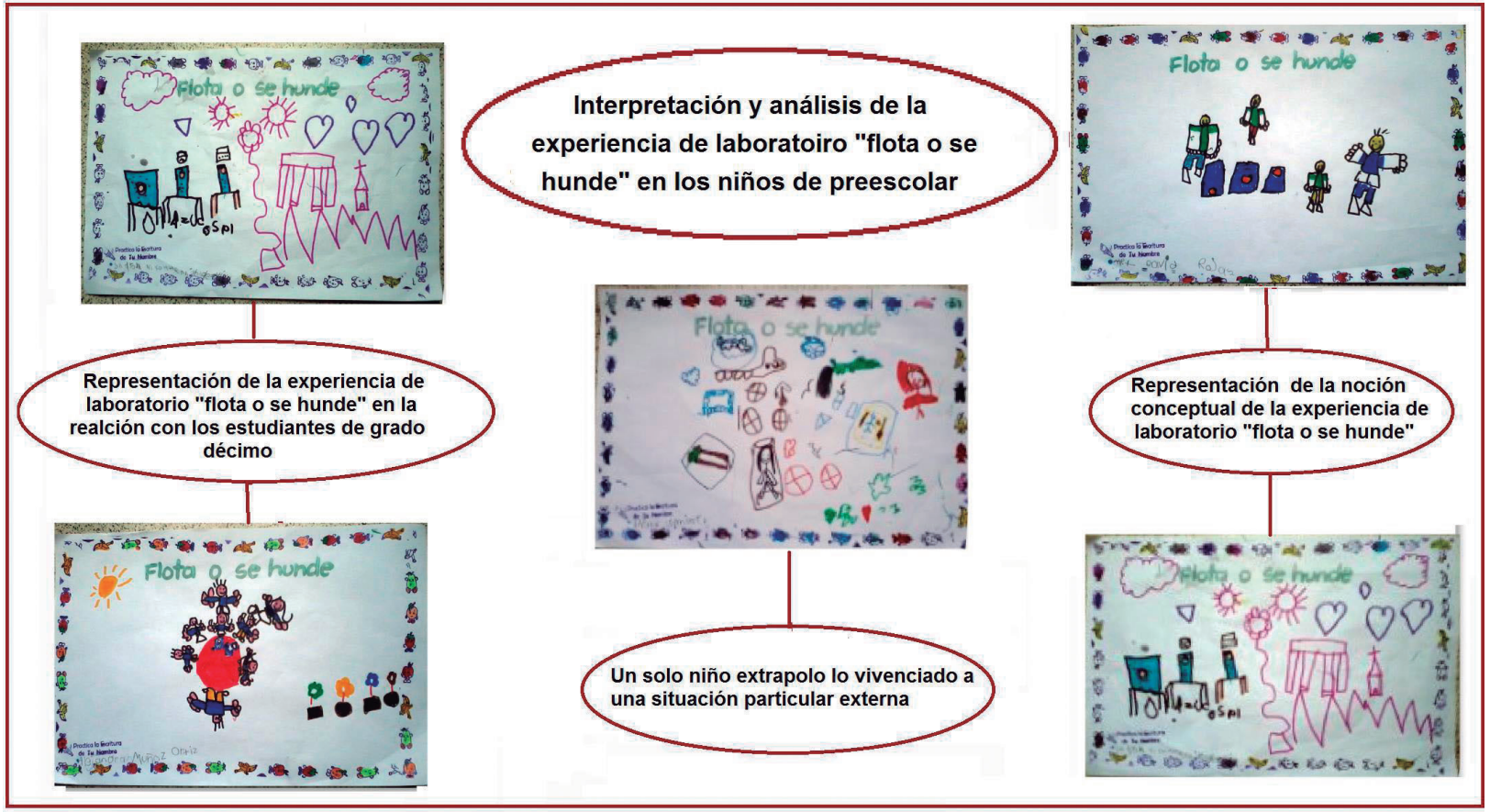

